\title{
Constraints on the Photon Mass from the Galactic Magnetic Field Structure
}

D. D. Ryutov

January 21,2010

Plasmas in the Laboratory and in the Universe: interactions, patterns, and turbulence COMO, Italy December 1, 2009 through December 4, 2009 
This document was prepared as an account of work sponsored by an agency of the United States government. Neither the United States government nor Lawrence Livermore National Security, LLC, nor any of their employees makes any warranty, expressed or implied, or assumes any legal liability or responsibility for the accuracy, completeness, or usefulness of any information, apparatus, product, or process disclosed, or represents that its use would not infringe privately owned rights. Reference herein to any specific commercial product, process, or service by trade name, trademark, manufacturer, or otherwise does not necessarily constitute or imply its endorsement, recommendation, or favoring by the United States government or Lawrence Livermore National Security, LLC. The views and opinions of authors expressed herein do not necessarily state or reflect those of the United States government or Lawrence Livermore National Security, LLC, and shall not be used for advertising or product endorsement purposes. 


\title{
Constraints on the Photon Mass from the Galactic Magnetic Field Structure
}

\author{
D.D. Ryutov \\ Lawrence Livermore National Laboratory \\ 7000 East Avenue, Livermore, CA 94551, USA
}

\begin{abstract}
The most efficient way to constrain the photon mass $\left(m_{p h}\right)$ is related to observations of largescale magnetic fields in space physics and astrophysics. This approach is based on the change in the Ampere law caused by the finite $m_{p h}$. In 1990s, a consistent set of MHD equations allowing for the finite $m_{p h}$ has been written and later used to analyze the solar wind data from the Voyager 1 and 2 missions. This lead to an estimate $m_{p h}<1.5 \times 10^{-51} \mathrm{~g}$, the value currently recommended by the bi-annual compendium of the Particle Data Group. The further progress in constraining the photon mass may come from considering the dynamics of large-scale magnetic fields in astrophysics, in particular, the magnetic field of galaxies. The paper is concerned with related opportunities and challenges, including the problem posed by the simultaneous presence of large-scale and much stronger small-scale magnetic fields. Effects of recycling of the interstellar plasma involving dense molecular clouds, protostars and supernovae explosions are discussed. Possible approaches to pushing the upper bound to a limit well below $10^{-51} \mathrm{~g}$ are discussed.
\end{abstract}

Keywords: Proca model, photon mass, galactic magnetic field PACS: 03.50De, 14.70.Bh, 96.50.Bh, 12.20.Fv

\section{INTRODUCTION}

The model of a massive photon was introduced in the modern physics in the 1930s, when so-called Proca equations have been suggested to describe a massive spin-1 particle [1]. Over the years, this model has been thoroughly tested for consistency and applied to a variety of physics problems (e.g., $[2,3])$. The photon mass $\left(\mathrm{m}_{\mathrm{ph}}\right)$ in this model is a parameter that has to be determined experimentally.

One of the first successful steps in this direction was made by E. Schroedinger [4] who noticed that the vector potential $\mathbf{A}$ of a magnetic dipole $\mathbf{m}$ falls off at large distances exponentially, in the Yukawa fashion: $\boldsymbol{A}=\left(\boldsymbol{\mu} / r^{2}\right) \exp (-r / \lambda)$, with $\lambda$ being the Compton length of a massive photon,

$$
\lambda=\hbar / m_{p h} c \text {, }
$$

and $c$ and $\hbar$ being the speed of light and the Planck constant, respectively. Throughout this paper we use CGS system of units. The "classical" Maxwell equations predict a power-law dependence, $A \sim 1 / r^{2}$, so that, at large enough distance from the source, the exponential term would lead to the observable deviations from the power-law scaling. As $\lambda$ is directly related to the photon mass, determining $\lambda$ is equivalent to determining the photon mass. Schroedinger applied this approach to the Earth magnetic field and could not see significant deviations at the distances $\sim$ the Earth radius. So, he could only constrain the Compton length from below, and, therefore, the photon mass from above, $m_{p h}<10^{-19} m_{e}$, with $m_{e}$ being the electron mass. Already this estimate has shown that it would be incredibly difficult to observe any effects of the finite photon mass in atomic or nuclear phenomena and also determined the strategy of the further attempts on improving 
(lowering) the upper bound. This strategy was the observation of magnetic field of various celestial bodies at large distances from them. A brief history of these earlier attempts is summarized in Ref. [5]. An insightful review of the photon mass issues was written recently by A. Goldhaber and M. Nieto [6].

Going to even greater scales means that one will have to account for the presence of the ambient plasma, which, generally speaking, carries non-negligible currents. Therefore, getting to larger scales makes it necessary to analyze a coupled system of the magnetic field and a plasma, and the dynamics of the conducting medium becomes an inseparable part of the problem, see, e.g., Refs. [7-10].

In the classical domain (as relevant to the subject of this paper), the finiteness of the photon mass manifests itself in the change of the Ampere law, which now becomes:

$$
\nabla \times B+\frac{A}{\hbar^{2}}=\frac{4 \pi}{c} j
$$

with $\boldsymbol{B}, \boldsymbol{A}$ and $\boldsymbol{j}$ being the magnetic field, the vector potential, and the current density, respectively, and $\boldsymbol{B}=\nabla \times \boldsymbol{A}$. We neglect the displacement current, as we consider only slow non-relativistic processes. Eq. (2) is a consequence of the Proca equations, describing a massive photon [1]; see also Refs. [2, 3, 6, 7]. The finite photon mass enters the problem via the second term in the left-hand side (1.h.s.) of Eq. (2): for the zero-mass photon $(\lambda \rightarrow \infty)$ we recover the standard Ampere law.

The expression for the ponderomotive force acting on the current-carrying medium remains the same as in zero-photon-mass electrodynamics

$$
\boldsymbol{f}=\boldsymbol{j} \times \boldsymbol{B} / c
$$

irrespectively to the possible finiteness of $m_{p h}[3,7]$. For the case where the length-scale $L$ is small compared to $\lambda$, one can neglect the second term in the left-hand side of Eq. (2), evaluate $j$ as $c B / 4 \pi L$ and obtain the "standard" estimate of the ponderomotive force,

$$
f \sim \frac{B^{2}}{8 \pi L} .
$$

Conversely, for the case where $L$ is large compared to $\lambda$, one has $j \sim c A / 4 \pi \lambda^{2}, A \sim B L$, so that the ponderomotive force increases significantly and becomes

$$
f \sim \frac{B^{2}}{8 \pi L}\left(\frac{L}{\lambda}\right)^{2} .
$$

The photon mass limit quoted in the latest edition of a bi-annual compendium of a Particle Data Group [11] was obtained in Ref. [5] by the analysis of the solar wind data at the Pluto orbit collected by the Voyager 1 and 2 missions. The approach used in Ref. [5] was as follows: Based on the knowledge of the global magnetic field of the Solar wind, one could evaluate its vector potential $\boldsymbol{A}$ and - via Eq. (2) - the current $\boldsymbol{j}$ required to sustain the observed magnetic field $\boldsymbol{B}$. This is particularly simple for the zone well 
beyond the Earth orbit, where the average $\boldsymbol{B}$ is almost entirely azimuthal. The latter was predicted in the original Parker model [12] and confirmed up to the distances of tens of astronomical units by a number of space missions [13-15]. The average flow in this zone is strongly supersonic and essentially radial, with the average velocity in the equatorial region $\mathrm{v}_{r} \approx 450 \mathrm{~km} / \mathrm{s}$ independent of the distance ("ballistic" flow).

For a large-enough photon mass (small-enough $\lambda$ ), the second term in the 1.h.s. of Eq. (2) becomes dominant, and $\boldsymbol{j}$ becomes much larger than in the case of a massless photon. This leads to the increase of the $\boldsymbol{j} \times \boldsymbol{B}$ force compared to the $m_{p h}=0$ case. For a large-enough value of $m_{p h}$ (small enough $\lambda$ ), deviations from the observed flow structure would become grossly incompatible with observations, thereby setting the upper bound on the photon mass. This then yielded an upper bound for $m_{p h}$ at the level of $m_{p h}<$ $1.5 \times 10^{-51} \mathrm{~g}[5]$.

This limit is based on direct in situ measurements and in this regard is as reliable as the limits established in the ground-based laboratory experiments in particle physics. The constraints of this type are sometimes called "hard" constraints [7] to contrast them to more speculative estimates not based on the observational data of the same level of details.

It is tempting to apply the same type of arguments to much larger systems, in particular, to tenuous magnetized interstellar plasmas in galaxies. The potential benefits should come from a large disparity of the "hard" limit on the Compton length $\lambda$ and a very large global scale $L$ of the problem. This point was made half a century ago by Yamaguchi [16] and then applied to Galactic magnetic field of the scale of $1 \mathrm{kpc}$ by Chibisov [17]. More recently, these arguments were repeated in Ref. [18], with a conclusion that the limit on the Compton length can be raised to $1 \mathrm{kpc}$ and the limit on the photon mass lowered, respectively, to $\sim 10^{-59} \mathrm{~g}$.

In this paper we discuss assumptions made on the way to this conclusions and find that the claimed limit cannot be considered as a "hard" limit, due to numerous uncertainties present in this problem. We try to identify a way to obtaining a "hard" (although much higher than $10^{-59} \mathrm{~g}$ ) estimate. In Sec. II we discuss an idealized model used before and describe a way in which the estimate of $10^{-59} \mathrm{~g}$ was obtained. In Sec. III we discuss the difficulties of an approach based on the virial theorem for the interstellar medium. In Sec. IV we present arguments in favor of obtaining a "hard" estimates by assessing the dynamics of relatively well characterized objects of the type of dense molecular clouds.

\section{AN IDEALIZED MODEL AND ITS DIFFICULTIES}

A simplest, strongly idealized model of a spiral arm would look as shown in Fig. 1a, an elongated plasma volume, with a quasi-uniform plasma and a quasi-uniform magnetic field directed along the larger dimension. Denoting the perpendicular size of the structure shown in Fig. 1 by $2 a$, one can make various assumptions regarding the ratio of $a / \lambda$ and consider the consequences.

One of the additional assumptions that one makes is that the system is in an "equipartition" state, i.e., the kinetic energy density is of order of the magnetic energy density and of the order of the total kinetic pressure $p$, that includes the pressure of a thermal gas and cosmic rays, 


$$
\rho \mathrm{v}^{2} \sim p \sim B^{2} / 8 \pi
$$

If one wants to consider the initial state as the state of equilibrium, one has to make some assumptions about the gravitational potential that keeps the gas from radial expansion. One can assume that the gravity is provided by the stellar material and, on larger scales, by the dark matter. In this model, the perpendicular dimension is set by the condition of hydrostatic equilibrium in the gravitational potential well, $p / a \sim \rho g$. This is a rough estimate, valid up to the factor of $2-3$, due to the fact that the equilibrium is affected also by the ram pressure and the magnetic pressure. Equipartition assumption (6) tells us also that $B^{2} / 8 \pi a \sim \rho g$.

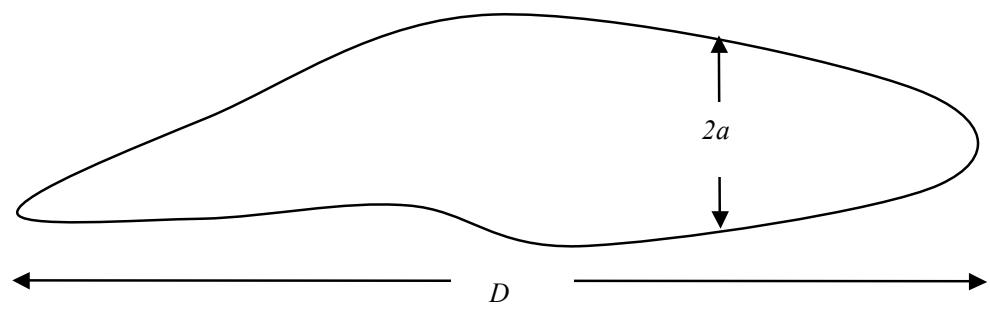

FIGURE 1. A sketch of an "isolated" piece of the spiral arm, with $2 a \sim 1 \mathrm{kpc}$, and $D \sim 10 \mathrm{kpc}$.

Assume now that the photon Compton length is significantly shorter than $a$. In this case, the $\boldsymbol{j} \times \boldsymbol{B}$ force, as was mentioned in the Introduction, becomes significantly larger than $B^{2} / 8 \pi a$ : according to Eq. (5), it becomes of order of $\left(B^{2} / 8 \pi a\right)\left(a^{2} / \lambda^{2}\right)$. Then, there is no way for the system shown in Fig. 1 to be in the mechanical equilibrium: with the equipartition assumption (6), the ponderomotive force (5) is much larger than all other forces involved. In other words, the system under consideration cannot be in an equilibrium. This consideration is then used as a proof that the condition $\lambda<a$ is incompatible with observations and, therefore, a condition

$$
\lambda>a
$$

must hold. For $a \sim 1 \mathrm{kpc}$, it yields exactly the limit $m_{p h}<10^{-59} \mathrm{~g}$.

This approach has, however, two flaws: 1) The interstellar medium is not an isolated dynamical system. It is continuously "recycled" by more massive and energyrich constituents of the spiral arm: supernova (SN) explosions, intense outflows from OB-type stars ("superbubbles"), condensation into dense molecular clouds, etc. 2) The magnetic field is grossly non-uniform, thereby making the estimate $A \sim B a$ questionable. For a review of the interstellar medium see Ref. [19]; for the magnetic field structure and the presence of smaller-scale fields see Refs. [20,21]. This more realistic (than Fig. 1) picture of the spiral arm is shown in Fig 2.

There is no reason whatsoever to think of the interstellar medium as an isolated system being in the state of a long-term equilibrium. Assume that we have identified a parcel of some size $L<a$, which is not affected by the aforementioned energetic processes and assume that $\lambda$ is significantly shorter than $L$. Then, indeed, fast flows on the scale of this parcel will ensue, with the acceleration of order of 


$$
\dot{\mathrm{v}} \sim f / \rho \sim \frac{B^{2}}{8 \pi \rho L}\left(\frac{L}{\bar{\lambda}}\right)^{2}
$$

The time for a significant deformation of the initial parcel will be

$$
\tau \sim \sqrt{L / \dot{\mathrm{v}}} \sim \frac{L}{\grave{\lambda}}\left(\frac{L}{\sqrt{B^{2} / 8 \pi \rho}}\right)
$$

In order this evolution to happen, there should not be major energetic events occurring in the volume under consideration for the time $\tau$. Take, for example, a parcel of the scale of $L \sim 300$ pc. Under the equipartition assumption (6) the Alfven velocity is of order of the sound speed in a $1 \mathrm{eV}$ hydrogen plasma, this meaning that the time $\tau$ will be of order of $\tau(y r) \sim 3 \cdot 10^{7}[300 / \lambda(p c)]$. On the other hand, the SN explosion in the parcel with the volume of $(300 \mathrm{pc})^{3}$ occurs, on average, once in $10^{5}$ year [19] and release energy comparable to or higher than the magnetic field energy in this volume. Constraining $\tau$ by $10^{5} \mathrm{yr}$, one finds that the limit on $\lambda$ becomes $\lambda>3 \mathrm{pc}$, this corresponding to the mass limit $m_{p h}<3 \cdot 10^{-57}$ g, i.e., 300 times softer than the "naïve" estimate (7). This example should not be considered as establishing a new, "realistic" upper bound; it just shows that the interstellar medium cannot be considered as an isolated system, is continuously raked by various energetic events, and the basic logic that has lead to Eq. (7) does not work.

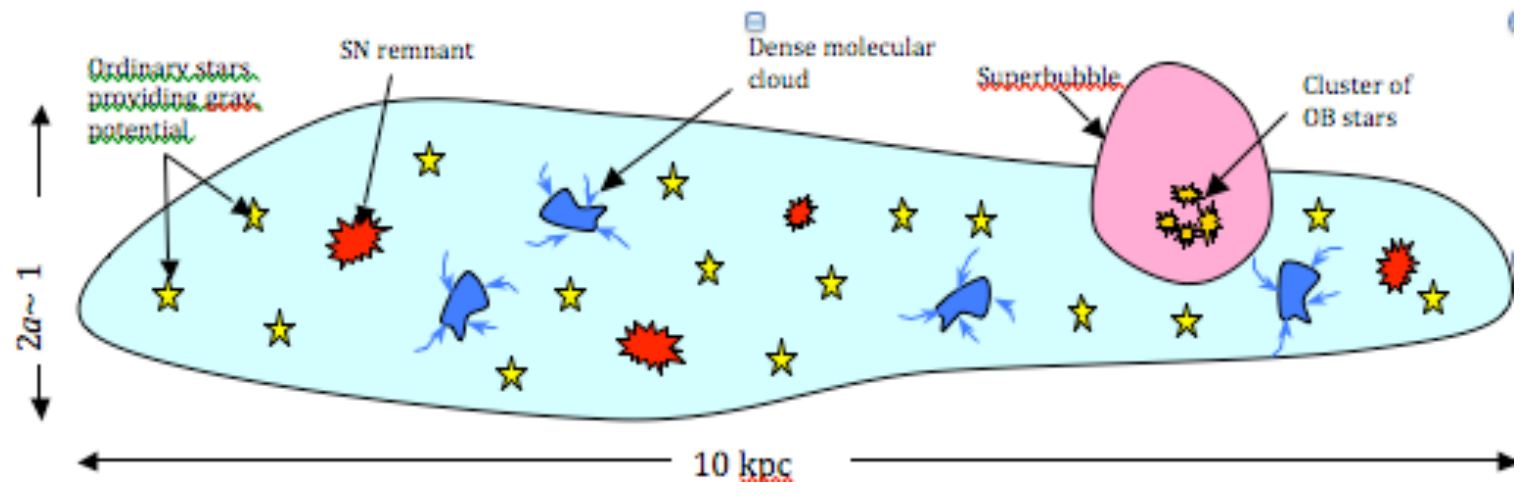

FIGURE 2. A segment of the spiral arm with the low-density interstellar medium shown in light-blue. Red irregular stars indicate supernova explosions, an yellow five-point stars indicate regular stars. Blue arrows indicate accumulation of mass by dense molecular clouds. Exchange of mass, momentum and energy between the interstellar medium (ISM) and other constituents of the spiral arm sets the time-scale within which the ISM can be considered as an isolated system.

\section{CAN ONE USE THE VIRIAL THEOREM?}

The same problem arises if one attempts to assess the behavior of the interstellar medium by means of the virial theorem, as was suggested in Ref. [17] and discussed in Ref. [18] (see Eqs. (17)-(18) of the latter paper). If one considers interstellar medium as an isolated system confined by the "averaged" gravity $\boldsymbol{g}$ of a stellar matter and, possibly, of the dark matter, the virial integral becomes 


$$
\int_{V}\left(\rho v^{2}+3 p+\frac{B^{2}}{4 \pi}\right) d V+\int_{V} \rho \boldsymbol{g} \cdot \boldsymbol{r} d V=\int_{V} \frac{A^{2}}{4 \pi \hbar^{2}} d V
$$

The main problem with it is, again, that we are dealing not with an isolated system, but with a system interacting with much more massive and energy-rich constituents of the Galaxy (for details see [22]). One cannot just balance different terms against one another and, from the lack of such a balance, come to some constraint on the photon Compton length.

The virial theorem reveals one more problem in the idealized approach of Sec. II. Specifically, this approach relies on the assumption that the magnetic field can be characterized by a single scale comparable to the global scale of the problem ( $a$ in Fig. 1). In reality, there is certainly strong random magnetic field with the scales much smaller than $a$. The r.m.s. value of this field is at least an order of magnitude higher than that of the average field. More details on that issue can be found in Refs. $[19,20]$.

With regard to a global magnetic field, the one that has a non-zero average over the scale $a$, one should be very cautious. Its determinations from the Faraday rotation of the polarized radiation of pulsars is based on a so-called rotation measure, which is proportional to the line-of-site integral of plasma density and the magnetic field. The higher density regions, therefore, introduce a bias in the field measurements [21]. One can even think of a situation where the average field is zero, but the rotation measure is non-zero [6]. Not trying to resolve this difficult problem, we consider possible effect of the presence, alongside with the average magnetic field $B$, also a much stronger fluctuating field $\tilde{B}>>B$. Let the spatial scale of this fluctuating field be $b<\lambda<<a$. Then, obviously, the right-hand-side of Eq. (10) will exceed the first term in the left-hand side and cause contraction of the plasma only if $\lambda<a(B / \tilde{B})$. In other words, we again obtain a constraint that is much weaker than (7).

\section{A “HARD” UPPER BOUND FROM ASTROPHYSICAL OBSERVATIONS}

In order to obtain a reliable constraint on $m_{p h}$ (or, equivalently, $\lambda$ ) from the behavior of interstellar medium, one needs to fulfill several conditions.

First, there should be information about the magnetic field and its structure. Important in this regard is the possible presence of multiple scales. The small scales can be critical in getting the correct upper bound.

Second, an area in the observational domain should be identified which is not affected by various energetic events, like SN explosions, massive outflows from young stars, or, conversely, condensation of the lower-density gas into denser molecular clouds.

Third, an observational signature of what would be different from the "normal" behavior (corresponding to a zero-mass photon) should be identified. An absence of such a signature would then mean that the photon mass is smaller than expected, this leading to a new upper bound.

As we have seen, the attempts to use the galactic magnetic field for constraining the photon mass have not met these requirements thus far. Significant uncertainties exist with the issue of the magnetic field structure; there are no clear predictions of what 
specific effects should be looked for. Also, energetic events make the isolation of purely ponderomotively-driven dynamics quite difficult (See Sec. II). This last point may favor moving away from the star-rich spiral arms to the inter-arm areas. On the other hand, these areas are also strongly affected by the effects like $\mathrm{SN}$ explosions and super-bubbles, which encompass areas well beyond the thickness of the galactic disk and inter-arm distances.

It might be worthwhile to focus on the galactic halo, where some magnetic field is present as well. On the other hand, the information about its structure is scarce, and the presence of a small-scale component is a distinct possibility.

Certainly, the very fact that the spatial scales of the galactic systems are incredibly large compared to the size of the Solar system supports an intuitive belief that the behavior of the gas in galaxies can lead to much stricter constraints than the presently accepted "hard" constraint on the photon mass. The author shares this belief but at present this new limit seems to be somewhat elusive.

To identify possible observational strategies, we present Fig. 3, where the evolution time (9) of a certain parcel of matter under the action of the Proca ponderomotive force (5) is shown for various assumptions regarding the ratio $L / \lambda$. We present the results as normalized to the Alfven crossing time $L / \mathrm{v}_{\mathrm{A}}$, with $\mathrm{v}_{A} \equiv B \sqrt{4 \pi \rho}$ being the "standard" Alfven velocity. The velocity reached by a matter within the parcel by the time $t$, when significant deformation of the packet occurs, is (see Eq. (4)) $\mathrm{v}=\mathrm{v}_{A}(L / \lambda)$. Note that, for small-enough $\lambda$, this velocity approaches the speed of light c. The parts of the plots corresponding to reaching relativistic velocities cannot be described by our analysis and are shown as dashed lines. The presence of these of this relativistic constraint can also be used to limit the photon mass, but one still needs to have a good information about the magnitude of the quasi-uniform magnetic field, the absence of small-scale random fields, and be able to identify the absence of any observational signatures of the predicted dynamics within the time $\tau$.

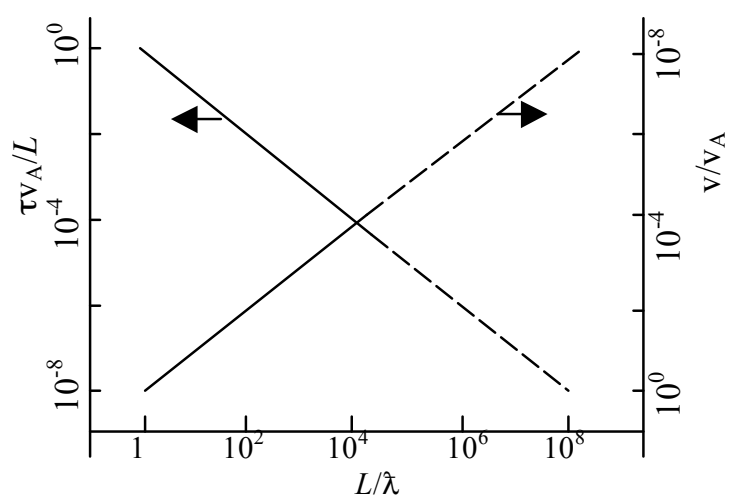

FIGURE 3. Evolution time $\tau$ (Eq. (9)) and the characteristic velocity $\mathrm{v}$ reached by the time $\tau$ vs the $L / \lambda$ ratio. The domain requiring relativistic description for $\mathrm{v}_{\mathrm{A}}=30 \mathrm{~km} / \mathrm{s}$ is shown by dotted lines.

Perhaps, the best chance to advance the "hard" limit is to look at some welldefined objects, like astrophysical protostellar jets or dense molecular clouds. In both cases the magnetic field seems to play a significant role in the overall dynamics and, at the same time, these objects are well localized and should not experience interference 
from other objects. As their scale is in the range of one parsec, one can hope to bring the "hard" limit to this value, $\lambda>1 p c$, or $m_{p h}<10^{-56} \mathrm{~g}$.

One more approach would be to apply the current "hard" estimate to the galactic system in the hope that, for large-enough photon mass, some phenomena would become wide-spread, observable in our Galaxy and other Galaxies as well. One such possibility is identifying hydrodynamic acceleration processes induced by strong domination of the Proca contribution (5) to the ponderomotive force (see Appendix). If the photon Compton length is indeed small, one can expect acceleration of collapsing parcels of gas to relativistic energies. For the presently accepted mass, and magnetic fields $\sim 1 \mu \mathrm{G}$ this would occur for the scales exceeding $\sim 1 \mathrm{pc}$.

\section{DISCUSSION}

Improving a "hard" upper bound on the photon mass, established from the in situ measurements of the Solar wind requires a knowledge of magnetic field structure in the range from $1 \mathrm{kpc}$ to a fraction of a pc. The possible presence of a significant small-scale random field complicates any attempts to find this better "hard" limit. The other complication comes from various energetic events that continuously "stir" the tenious interstellar plasma. Some progress can possibly be made by analyzing the dynamics of well-localized objects like protostellar jets and dense molecular clouds, but this would require better knowledge of the magnetic field present in these objects.

\section{ACKNOWLEDGMENT}

This work was performed under the auspices of the U.S. Department of Energy at Lawrence Livermore National Laboratory under Contract DE-AC52-07NA27344.

\section{APPENDIX: 1D EQUATIONS OF PROCA MAGNETOHYDRODYNAMICS}

It is instructive to provide a brief analysis of 1D motion for the Proca magnetohydrodynamics [10]. Assuming that the variation occurs in the $x$-direction, the magnetic field is along $z$, and vector potential is along $y$, one obtains, for the perfectly conducting medium, the following set:

$$
\begin{aligned}
& \rho \frac{d \mathrm{v}}{d t}=-\frac{\partial p}{\partial x}-\frac{1}{8 \pi} \frac{\partial}{\partial x}\left(B^{2}-\frac{A^{2}}{\lambda^{2}}\right) \\
& B=\frac{\partial A}{\partial x} ; \frac{\partial B}{\partial t}+\frac{\partial}{\partial x}(B \mathrm{v})=0 \\
& \frac{\partial \rho}{\partial t}+\frac{\partial}{\partial x}(\rho \mathrm{v})=0 ; \rho \frac{d p}{d t}-\gamma p \frac{d \rho}{d t}=0
\end{aligned}
$$


where $\mathrm{v}$ stands for the $\mathrm{x}$-component of the velocity. Assuming that the vector potential at large $|x|$ tends to zero (as required by the finiteness of the total energy), one sees that the line-tying equation leads to the following equation for $A: \mathrm{d} A / \mathrm{dt}=0$, i.e., the vector potential is advected together with the fluid element. This observation allows one to conclude that, for the system with the scale-length. The presented set of equations possesses the energy integral,

$$
\int d x\left(\frac{\rho v^{2}}{2}+\frac{B^{2}}{8 \pi}-\frac{A^{2}}{8 \pi \lambda^{2}}+\frac{p}{\gamma-1}\right)=\text { const }
$$

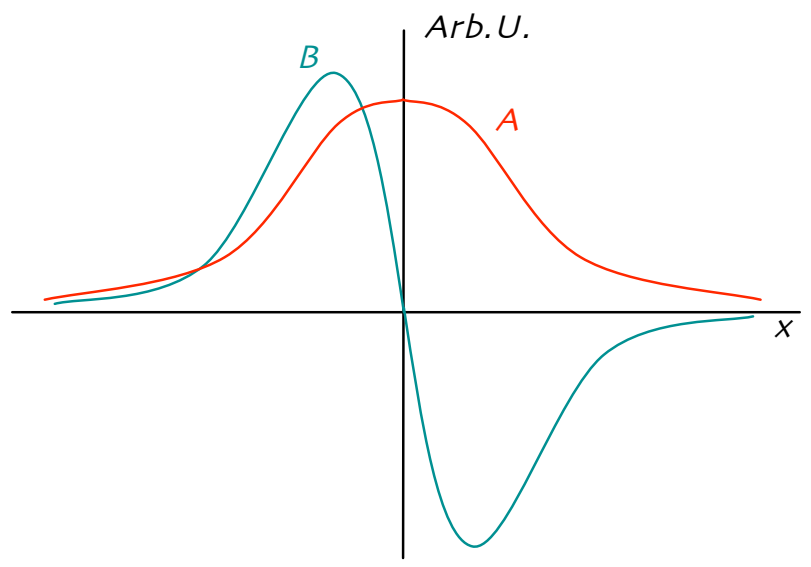

FIGURE 4. Initial distribution of $B$ and $A$ discussed in the Appendix.

If one starts from the distribution of various quantities as shown in Fig. 4, with a strong domination of the ponderomotive force associated with the vector potential, one can neglect the pressure and magnetic field terms in the dynamic equations. Initially, there will occur an acceleration of the gas with its strong compression, but eventually the magnetic pressure takes over and causes a rebound. For small enough $\lambda$, the motion near the rebound point would become relativistic (not described by our equations). The absence of such occurrences might serve as a way for establishing a better constraint on the photon mass. On the other hand, if the photon mass was large-enough, these areas of a relativistic acceleration could be widely spread in the Universe and serve as one more candidate for cosmic ray generation. 


\section{REFERENCES}

1. A. Proca, "Sur l'equation de Dirac," Compt. Rend. 1901377 (1930).

2. L.D. Landau, E.M. Lifshitz, Quantum Theory of Fields (Oxford, Pergamon), 1987.

3. J.D. Jackson, Classical Electrodynamics (Chichester, Wiley), 1975.

4. E. Schroedinger, "The Earth's and the Sun's Permanent Magnetic Fields in the Unitary Field Theory," Proc. Royal Irish Academy A49, 135 (1943).

5. D.D. Ryutov. "Using Plasma Physics to Weigh the Photon." Plasma Phys. Contr. Fus., 49, B429, 2007.

6. A.S. Goldhaber, M.M. Nieto. "Photon and Graviton Mass Limits". To appear in Rev. Mod. Phys, 2010.

7. A.S. Goldhaber, M.M. Nieto, "Terrestrial and Extraterrestrial Limits on the Photon Mass," Rev. Mod. Phys., 43, 277 (1971).

8. M.A. Gintzburg, Soviet Astronomy, AJ7, 536 (1964).

9. J.V. Hollweg, Phys. Rev. Lett., 32, 961 (1974).

10. D.D. Ryutov "The role of finite photon mass in magnetohydrodynamics of space plasmas," Plasma Phys. Control. Fusion 39, A73 (1997).

11. C. Amsler, and Particle Data Group, Phys. Letters, B667, 1, 2008.

12. E.N. Parker. Astrophys. Journ. 128, 664, 1958.

13. L.F. Burlaga, N.F. Ness, Y-M. Wang, N.R. Sheeley. Journ. Geophys. Res. 103 23723, 1998.

14. N.F. Ness, L.F. Burlaga. Journ. Geophys. Res. 106 \#A8, 15803, 2001.

15. E.J. Smith. Solar wind magnetic fields. In: "Cosmic Winds and heliosphere,” p. 425, J.R. Jokipi, C.P.

Sonett, M.S. Giampapa, Eds., The University of Arizona Press, Tucson,1997.

16. Y. Yamaguchi, "A composite theory of elementary particles," Progr. Theor. Phys. \#11, 1 (1959).

17. G.V. Chibisov, "Astrophysical upper limit on the photon rest mass," Sov. Phys. Uspekhi 19, (1976) 624.

18 E. Adelberger, G. Dvali, A. Gruzinov, "Photon mass destroyed by vortices," Phys. Rev. Lett. 98, 010402 (2007).

19. K.M. Ferriere, "The interstellar environment of our galaxy," Rev. Mod. Phys. 731031 (2001).

20. Han J-L 2006 The Galactic magnetic field Journ. of Phys. Conf. Series 47120.

21. Beck R, Shukurov A, Sokoloff D, Wielebinski R 2003 Systematic bias in interstellar magnetic field estimates Astron. \& Astrophys. 41199.

22. D.D. Ryutov. "The virial theorem for the interstellar medium Astrophys. Journ. 674, 976 (2008).

23. D.D. Ryutov. "Relating the Proca photon mass and cosmic vector-potential via solar wind." Phys. Rev. Lett. 103, 201803 (2009). 